\title{
Types of Balkan butterflies in the collection of Muséum national d'Histoire naturelle, Paris (Lepidoptera: Papilionoidea)
}

\author{
Stanislav Abadjiev
}

\begin{abstract}
National Museum of Natural History, Bulgarian Academy of Sciences, 1 Tsar Osvoboditel Blvd, 1000 Sofia, Bulgaria, abadjiev@nmnhs.com
\end{abstract}

http://zoobank.org/F8E9B406-0D08-4C8D-B60F-5CD2899F07E5

\begin{abstract}
The list comprises type material of butterflies from the Balkan Peninsula kept at Laboratoire d'Entomologie, Muséum national d'Histoire naturelle, Paris. The collection contains types of 26 nominal species group taxa, described primarily by Hans Fruhstorfer, but also by Hans Rebel, Felix Bryk, Curt Eisner, Georges Bernardi. Six taxa belong to the family Papilionidae, one to Pieridae, and 19 to Nymphalidae. For the stability of nomenclature, lectotype has been designated for Euphydryas maturna idunides (Fruhstorfer, 1917).
\end{abstract}

Keywords: Balkan Peninsula, holotype, lectotype, Nymphalidae, Papilionidae, Pieridae, syntype

\section{Introduction}

Taxonomic studies of any group of animals, including butterflies, Papilionoidea, serve as a basis for future investigations. Of special importance in any taxonomic study is the good knowledge on the type material of the taxa in question, and as far as name-bearing types (holotypes, lectotypes, syntypes) serve as a standard of nominal species group taxa, it is important to know all the data which can help in their subsequent recognition (including labels descriptions). Moreover, publishing lists of such types housed at certain institutions is even recommended (ICZN, 1999: 72F.4).

The Muséum national d'Histoire naturelle in Paris (Fig. 1) is well known as a depository of important collection of type material of World Lepidoptera, with many taxa represented by primary types. The butterfly collection kept at Laboratoire d'Entomologie occupies several rooms and is housed in different types of shelves and cabinets.

The catalogue presented here continues the series on type material of Balkan butterflies already published - of the former Institute of Zoology [Institute of Biodiversity and Ecosystem Research] at the Bulgarian Academy of Sciences, Sofia (Abadjiev, 2001) and The Natural History Museum, London (Abadjiev,
2002, 2006). For the purpose of the current work, the study area has been expanded outside the geographical boundaries of the Balkan Peninsula and includes all the territory of the former Yugoslavia and some Greek islands.

$\begin{array}{ll}\text { Abbreviations } \\ \text { BMNH } & \begin{array}{l}\text { The Natural History Museum, London } \\ \text { ibidem [in that very place] }\end{array} \\ \text { ibid. } & \begin{array}{l}\text { International Commission on Zoological } \\ \text { ICZN }\end{array} \\ \text { MNHN } & \begin{array}{l}\text { Nomenclature } \\ \text { Muséum national d'Histoire naturelle, }\end{array} \\ \text { ZMHB } & \begin{array}{l}\text { Paris } \\ \text { Mniversität, Berlin }\end{array}\end{array}$

\section{Results and discussion}

This publication is a result of my research visit to the Laboratoire d'Entomologie, Muséum national d'Histoire naturelle, Paris in October 2003. A revision of the collection there allowed to find Balkan butterfly type material represented by 113 specimens belong- 
ing to 26 nominal species group taxa. The collection contains types of taxa described primarily by the German explorer, insect trader and entomologist Hans Fruhstorfer (1866-1922), but also by the Austrian entomologist Hans Rebel (1861-1940), the Swedish anthropologist, entomologist and writer Felix Bryk (1882-1957), the German entomologist Curt Eisner (1890-1981), and the French entomologist Georges Bernardi (1922-1999).

The names of the taxa are arranged in alphabetic order and each entry includes the associated family name, original combination quoted from the original publication, type locality (containing a quotation from the original publication or subsequent fixation due to lectotype designation), type specimens as specified with their labels, and notes about the type material and/or current taxonomic status. All extensions of used abbreviations or remarks are enclosed in square brackets "[ ]". The text of the label is quoted in double quotation marks and each line in the text of the label is separated by a vertical line ""); in quotations of combined labels (handwritten on printed forms) the handwritten text is reproduced in italics, completely handwritten and completely printed labels are quoted in a plain character face.

boris Fruhstorfer, 1917 (Nymphalidae) - "Melitaea athalia boris subspec. nova" (Fruhstorfer, 1917b: 19). Type locality: "Bulgarien, Rhodope [Rila]" (ibid.: 19). Holotype 9 (by monotypy) with labels: (1) printed with handwritten inscriptions, here italicised (on white paper) "Bulg. Rhodope Geb. I 11.7 | M. Hilf 1911 | Coll. O. Leonhard"; (2) printed (on red paper) "Type"; (3) printed (on white paper) "MUSÉUM PARIS | 1934 | COLL H FRUHSTORFER"; (4) printed with handwritten inscriptions, here italicised (on white paper) "MUSÉUM PARIS | figuré | par |Verity"; (5) handwritten (on white paper) "Melitaea athalia $\mid$ ssp. boris Frhst. $\mid 1917$ (Soc. Ent. p. 19| et 180)"; (6) printed (on white paper) "MNHN $|0031|$ $\mathrm{S}$. Abadjiev". The specimen is collected in the current territory of Rila Mountains, as with all the material of Moritz Hilf. At that time its eastern part was considered part of the Rhodopes. A junior subjective synonym of Papilio athalia athalia Rottemburg, 1775.

bosna Fruhstorfer, 1908 (Nymphalidae) - "Argynnis amathusia bosna nov. subspec." (Fruhstorfer, 1908b: 214). Type locality: "Bosnien, Trebevic... Sarajevo und Hodza" (ibid.: 214). Syntypes $7 \lesssim \widehat{\jmath}$ with labels: [1] (1) printed (on white paper) "amathusia | bosna | Fruhst."; (2) printed (on white paper) "MNHN

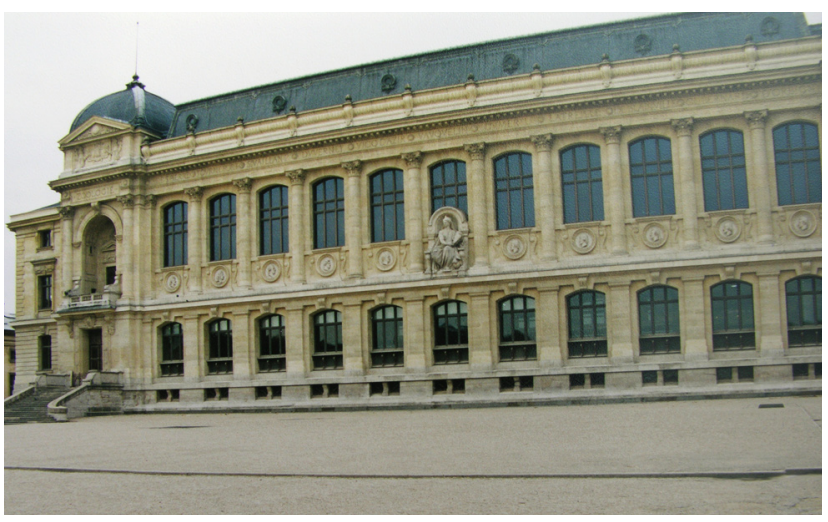

Fig. 1. The emblematic building of the Grande galerie de l'Évolution of the Muséum national d'Histoire naturelle, Paris (photo: S. Abadjiev, October 2003).

| 0031 | S. Abadjiev"; [2] printed (on white paper) "MNHN | 0046 | S. Abadjiev"; [3] printed (on white paper) "MNHN | 0047 | S. Abadjiev"; [4] (1) printed with handwritten inscriptions, here italicised (on white paper with black frame) "Wettl | Hodža | 16.6.08"; (2) printed (on white paper) "MNHN | $0048 \mid$ S. Abadjiev"; [5] printed (on white paper) "MNHN | 0049 | S. Abadjiev"; [6] printed (on white paper) "MNHN | 0050 | S. Abadjiev"; [7] (1) printed (on white paper with black frame) "Wettl | Trebevic | 5.7.08"; (2) printed (on white paper) "MUSÉUM PARIS | 1969 | Coll. Dr. P. Acheray"; (3) printed (on white paper) "MNHN | 0051 | S. Abadjiev"; syntypes 1-3, 5, 6 with additional: printed (on white paper) "Bosnia $\mid$ Trebevic 8.7.06 | DR. SCHAWERDA"; syntypes 1-6 with additional: printed (on white paper) "MUSÉUM PARIS | 1934 | COLL H FRUHSTORFER"; syntypes 2-7 with additional: printed (on white paper) "SYNTYPE of | bosna | Fruhstorfer, | 1908". Originally the type series comprises $10 \hat{\partial} \hat{\partial}$ (ibid.: 214). Infrasubspecific taxon; belongs to Boloria titania (Esper, 1793).

bulgarica Eisner, 1936 (Papilionidae) "P[arnassius $]$. mnemosyne subsp. bulgarica (subsp. nova)" (Eisner, 1936: 9). Type locality: "Pisanec am Lom [Pisanets Village, Rusenski Lom Nature Park], 300 m N.O. Bulgarien" (ibid.: 9). Paralectotype $\widehat{\sigma}$ with labels: (1) printed (on white paper) "Pisanec am Lom | N.O. Bulgarien 300m. | V.1935 leg.Heinrich"; (2) printed (on red paper) "PARATYPE"; (3) printed (on white paper) "MUSÉUM PARIS"; (4) handwritten (on white paper with black frame) "subsp. psyche | Eisner"; (5) printed (on white paper with black frame) "Coll. Eisner | Berlin"; (6) handwritten (on white pa- 
per) "subsp. | bulgarica | Eisner, 1936", on the back "S. Abadjiev | 2003"; (7) printed (on white paper) "MNHN | 0017 | S. Abadjiev". Originally the type series comprises $19 \hat{\jmath} \widehat{\partial}, 18$ 우 (ibid.: 9). Lectotype designated by Eisner (1966: 10). Additional paralectotypes 3 ते, 1 q in BMNH (Abadjiev, 2002: 13). A junior subjective synonym of Parnassius mnemosyne caucasia Verity, [1911] (Abadjiev, 2002: 13).

bulgarica Fruhstorfer, 1917 (Nymphalidae) "M[elitaea $]$. aurinia bulgarica subspec. nova" (Fruhstorfer, 1917a: 6). Type locality: "Bulgarien, Rila, 1700 m" (ibid.: 6). Lectotype $\hat{O}$, paralectotypes 2 $\hat{\jmath} \hat{\partial}, 1$ + with labels: [1 $\hat{\partial}$, lectotype] (1) printed with handwritten inscriptions, here italicised (on white paper with black frame) "Bulgarien $\mid \mathrm{Al}$. K. Drenowski”; (2) printed (on white paper with black frame) "Rilaplanina | $1700 \mathrm{~m}$ ", on the back "26.VII. | 1906"; (3) printed (on red paper) "Type"; (4) printed (on white

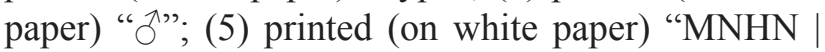
0034 | S. Abadjiev"; [2 3 ] printed (on white paper)

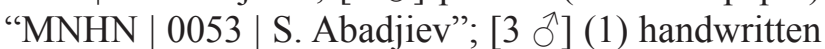
(on white paper with black frame) "aurinia | bulgarica | Fruhst."; (2) printed (on white paper) "MNHN | 0054 | S. Abadjiev"; [4 9 ] printed (on white paper) "MNHN | 0035 | S. Abadjiev"; all the types with additional: printed (on white paper) "MUSÉUM PARIS | 1934 | COLL H FRUHSTORFER"; paralectotypes 2-4 with additional: printed with handwritten inscriptions, here italicised (on white paper) "Bulg. Rhodope Geb. | 30.7 | M. Hilf 1911 | Coll. O. Leonhard”; lectotype and paralectotype 4 with additional: handwritten (on white paper) "Melitaea aurinia $\mid$ ssp. bulgarica Frhst. 1916 (Arch. Nat. A, | 2, p. 6)". Originally the type series comprises exactly $3 \hat{\partial} \widehat{\partial}, 1$ ( ibid.: 6). Although not labelled properly, lectotype has been designated by Bernardi \& de Lesse (1951: 143). Treated as a distinct subspecies, Euphydryas aurinia bulgarica (Fruhstorfer, 1917) (Hesselbarth at al., 1995: 1020).

dardanus Rebel, 1917 (Papilionidae) - "Parnassius apollo (L.) dardanus n. subsp." (Rebel, 1917: 773). Type locality: "Zljeb... in Höhen zwischen 1500 und 1750 m... Vunsaj" (ibid.: 773). Syntypes 5 $\widehat{\partial}$ with labels: [1] (1) printed with handwritten inscriptions, here italicised (on white paper) "NeuMonteneg. |Zljeb Penth '16 | 11. Juli'; (2) printed (on white paper) "MNHN | $0011 \mid$ S. Abadjiev"; [2] (1) printed with handwritten inscriptions, here italicised (on white paper) "Neu-Monteneg. | Zljeb Penth'16 | 16.VII"; (2) printed (on white paper) "MNHN $0012 \mid$ S. Abadjiev"; [3] (1) printed with handwritten inscrip- tions, here italicised (on white paper) "Neu-Monteneg. | Zljeb Penth '16 | 18.VII'; (2) handwritten (on white paper) "P. apollo L. | dardanus $\mid$ cotype [in red] | Rbl."; (3) printed (on white paper) "Dr. H. Kolar dedit 1925"; (4) printed (on white paper) "MNHN 0013 | S. Abadjiev"; [4] (1) printed with handwritten inscriptions, here italicised (on white paper) "NeuMonteneg. | Zljeb Penth '16 | 14.VII'; (2) printed (on white paper) "MNHN | $0014 \mid$ S. Abadjiev"; [5] (1) printed with handwritten inscriptions, here italicised (on white paper) "Neu-Monteneg. | Zljeb Penth'16| 4.VII"; (2) handwritten (on white paper) "dardanus | Rebel", on the back "akbesiana"; (3) printed (on white paper) "MNHN | 0015 | S. Abadjiev"; all the syntypes with additional: printed (on white paper) "MUSÉUM PARIS | 1952 | COLL R OBERTHÜR"; syntypes 1, 2 with additional: handwritten (on white paper) "P. apollo L. | dardanus [in red] | Type [in red] Rbl.”. Originally the type series comprised more than $24 \hat{\partial} \partial^{\lambda}, 5$

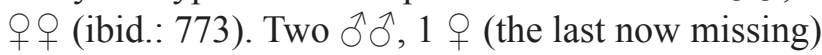
mentioned by Bernardi \& Viette (1966: 229).

dinara Fruhstorfer, 1908 (Nymphalidae) - "Argynnis amathusia dinara nov. subspec." (Fruhstorfer, 1908b: 214). Type locality: "Herzegowina, Gacko... Vucija-bara" (ibid.: 214). Syntypes 1 $\delta, 3$ 우 with labels: [1 $\left.\oint^{\Uparrow}\right]$ (1) printed (on white paper) "amathusia | dinara |Fruhst."; (2) printed (on white paper) "MNHN | 0040 | S. Abadjiev"; [2 +] printed (on white paper) "MNHN | 0041 | S. Abadjiev"; [3 9 ] printed (on white paper) "MNHN | 0044 | S. Abadjiev"; [4 9] (1) printed (on white paper with black frame) "Hercegowina Vucija bara | 10. Juli 08 | Dr. Schawerda"; (2) printed (on white paper) "MNHN | 0045 | S. Abadjiev"; all the syntypes with additional: printed (on white paper) "MUSÉUM PARIS | 1934 | COLL H FRUHSTORFER"; syntypes 1-3 with additional: printed (on white paper) "Hercegovina | Gacko | F. v. Meissl"; syntypes 1, 2 with additional: printed (on red paper) "Type"; syntypes 3, 4 with additional: handwritten (on white paper) "SYNTYPE $q$ | dinara | Fruhstorfer, | 1908 | S. Abadjiev, 2003". Originally the type series comprises exactly 10,3 우 (ibid.: 214). Infrasubspecific taxon; belongs to Boloria titania (Esper, 1793).

drenowskyi Bryk \& Eisner, 1934 (Papilionidae) - "[Parnassius mnemosyne] subsp. drenowskyi (subsp. nova)" (Bryk \& Eisner, 1934: 9). Type locality: "[Bulgaria], Varna, Kl. Galata" (ibid.: 9). Paratype $\widehat{\partial}$ with labels: (1) handwritten (on white paper) "Bulgaria | Galata | 20/5-31"; (2) printed (on red paper) "PARATYPUS"; (3) printed (on white paper) "MU- 

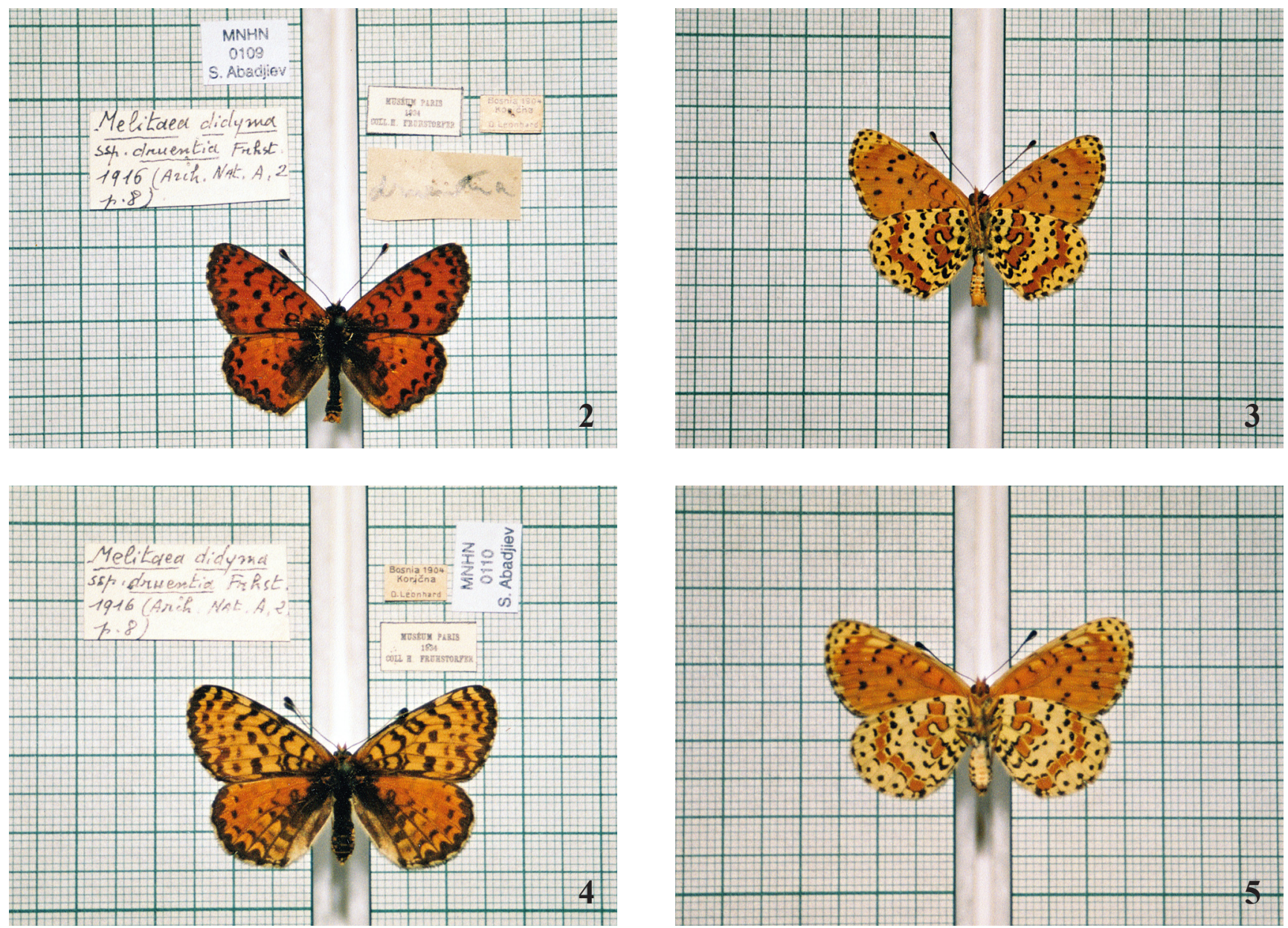

Figs 2-5. "Melitaea didyma druentia" Fruhstorfer, 1917 from Bosnia and Herzegovina: [2] lectotype ふ઼; [3] ditto, underside; [4] paralectotype $O$; [5] ditto, underside.

SÉUM PARIS"; (4) handwritten (on white paper with black frame) "subsp. drenowskyi | Bryk \& Eisner"; (5) printed (on white paper with black frame) "Coll. Eisner | Berlin"; (6) printed (on white paper) "MNHN | 0016 | S. Abadjiev". Originally the type series comprises $6 \hat{\jmath}, 4$ 우 (ibid.: 9). The specimen has been mentioned by Bernardi \& Viette (1966: 95).

druentia Fruhstorfer, 1917 (Nymphalidae) "M[elitaea]. didyma druentia subspec. nova" (Fruhstorfer, 1917a: 8). Type locality: "Bosnien, Coricna, Maklenpaß... Doboj" (ibid.: 8). Lectotype §ิ totypes $1 \hat{\jmath}, 2$ 우 with labels: [1 $\hat{\jmath}]$ (1) printed with handwritten inscriptions, here italicised (on white paper, framed) "Bosnia 1902 | Maklen Pass | O. Leonhard | 24.6."; (2) handwritten (on white paper with black frame) "M. didima | ad graceam"; (3) printed (on white paper) "MNHN | 0079 | S. Abadjiev"; [2 ડ̄, lectotype] (Figs 2-3) (1) handwritten (on white paper) "druentia"; (2) handwritten (on white paper) "Melitaea didyma $\mid$ ssp. druentia Frhst. | 1916 (Arch. Nat. A, 2 | p. 8)"; (3) printed (on white paper) "MNHN | 0109 | S. Abadjiev"; [3 q, without abdomen] printed (on white paper) "MNHN | 0080 | S. Abadjiev"; [4 9 ] (Figs 4-5) (1) handwritten (on white paper) "Melitaea didyma | ssp. druentia Frhst. | 1916 (Arch. Nat. A, 2, | p. 8)"; (2) printed (on white paper) "MNHN | 0110 | S. Abadjiev"; all the types with additional: printed (on white paper) "MUSÉUM PARIS | 1934 | COLL H FRUHSTORFER"; lectotype and paralectotypes 3 , 4 with additional: printed (on white paper) "Bosnia 1904 | Korična | O. Leonhard". Although not labelled properly, lectotype designated by Bernardi \& de Lesse (1951: 139). A junior subjective synonym of Papilio didyma didyma Esper, [1778] (Higgins, 1941: 216).

idunides Fruhstorfer, 1917 (Nymphalidae) "M[elitaea]. maturna idunides subspec. nova" (Fruh- 
Types of Balkan butterflies in the collection of Muséum national d'Histoire naturelle, Paris
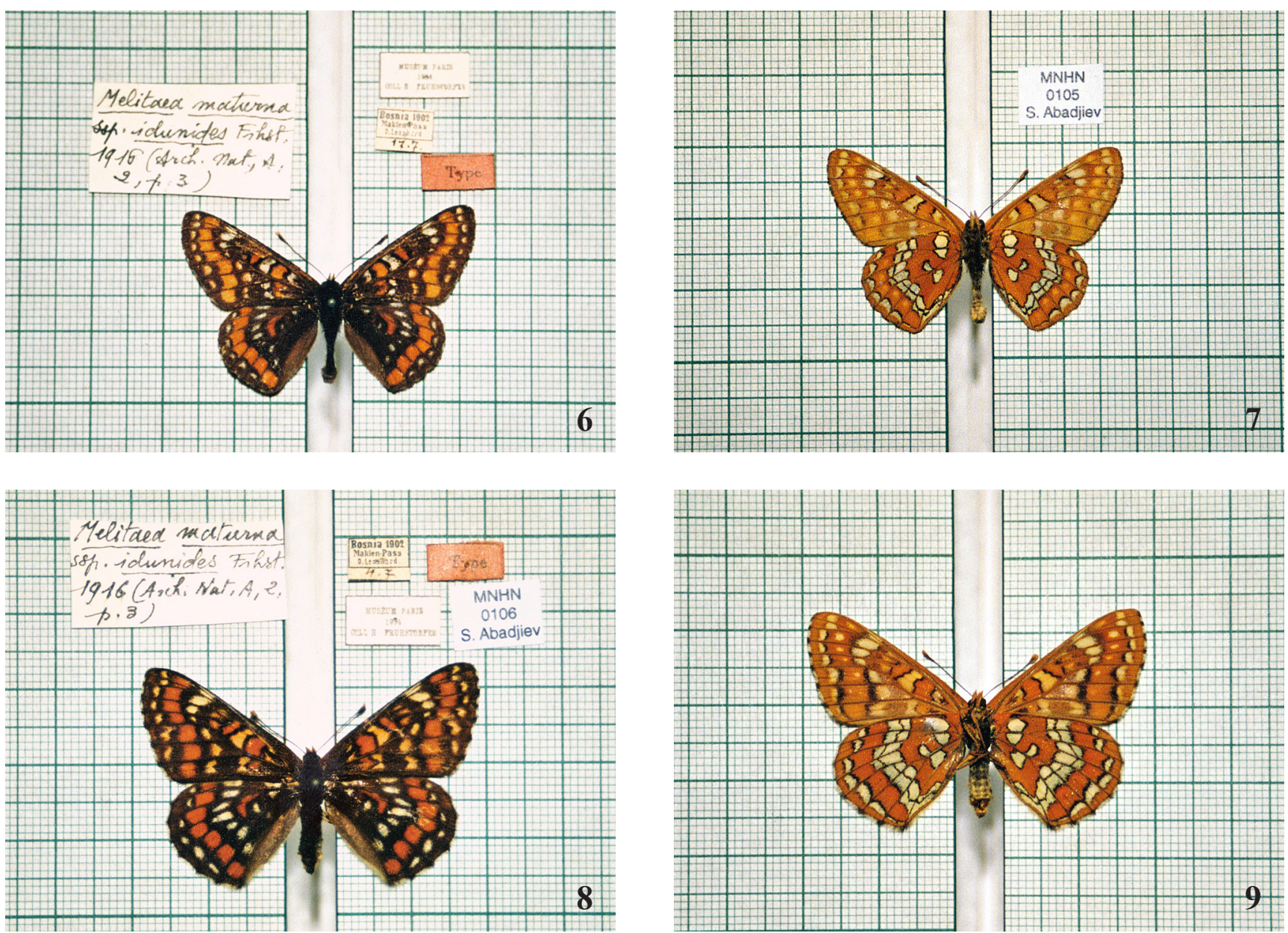

Figs 6-9. Euphydryas maturna idunides (Fruhstorfer, 1917) from Bosnia and Herzegovina: [6] lectotype $\overbrace{}^{\lambda}$; [7] ditto, underside; [8] paralectotype ${ }_{+}$; [9] ditto, underside.

storfer, 1917a: 3). Type locality: "Bosnien, Maklenpaß" (ibid.: 3). Lectotype $\widehat{\jmath}$ (here designated), paralectotypes $1 \hat{\jmath}, 2$ 우 with labels: [1 $\left.\begin{array}{l}1 \\ 0\end{array}\right]$ printed (on white paper) "MNHN | 0078 | S. Abadjiev"; [2 ふૈ, lectotype] (Figs 6-7) (1) handwritten (on white paper) "Melitaea maturna | ssp. idunides Frhst. | 1916 (Arch. Nat., A, | 2, p. 3)"; (2) printed (on white paper) "MNHN | 0105 | S. Abadjiev"; [3 O] (1) printed with handwritten inscriptions, here italicised (on white paper, framed) "Bosnia 1902 | Maklen Pass | O. Leonhard | 19.7."; (2) printed (on white paper) "MNHN | 0077 | S. Abadjiev"; [4 9] (Figs 8-9) (1) printed with handwritten inscriptions, here italicised (on white paper, framed) "Bosnia 1902 | Maklen Pass | O. Leonhard |4.7."; (2) handwritten (on white paper) "Meli-

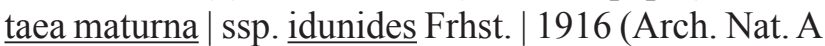
2, | p. 3)"; (3) printed (on white paper) "MNHN | 0106 | S. Abadjiev"; all the types with additional: printed (on white paper) "MUSÉUM PARIS | 1934 | COLL H FRUHSTORFER"; paralectotype 1, lectotype with additional: printed with handwritten inscriptions, here italicised (on white paper, framed) "Bosnia 1902 | Maklen Pass | O. Leonhard | 17.7."; paralectotypes 1, 3 with additional: handwritten (on white paper with black frame) "maturna | idunides | Fruhst,"; lectotype, paralectotype 4 with additional: printed (on red paper) "Type". Originally the type series comprises more than 3 ธิธ, 3 oq (ibid.: 3). The lectotype designation by Bernardi \& de Lesse (1951: 143) is considered invalid since there are 2 specimens labelled "Bosnia 1902 | Maklen Pass | O. Leonhard | 17.7." and none of them is labelled properly as a lectotype. Lectotype designation here was done for the stability of nomenclature. Treated as a distinct subspecies, Euphydryas maturna idunides (Fruhstorfer, 1917) (Rákosy et al., 2012: 146). 
lathon Fruhstorfer, 1917 (Nymphalidae) "M[elitaea]. trivia lathon subspec. nova" (Fruhstorfer, 1917a: 13). Type locality: "Velebit, Bosnien, Coricna" (ibid.: 13). Lectotype $\widehat{O}$, paralectotypes 3 우 with labels: [1 ${ }^{\lambda}$, lectotype] (Figs 10-11) (1) handwritten (on white paper) "Melitaea trivia | ssp. lathon Frhst. | 1916 (Arch. Nat., | A, 2, p. 13)"; (2) printed with handwritten inscriptions, here italicised (on white paper with black frame) "trivia $\mid$ lathon $\mid$ Fr."; (3) printed (on white paper) "MNHN | $0112 \mid$ S. Abadjiev"; [2 9] printed (on white paper) "MNHN | $0075 \mid$ S. Abadjiev"; [3 9] printed (on white paper) "MNHN | $0076 \mid$ S. Abadjiev"; [4 + ] (1) handwritten (on white paper) "Melitaea trivia | ssp. lathon Frhst. | 1916 (Arch. Nat., A, | 2, p. 13)"; (2) printed (on white paper) "MNHN | 0111 | S. Abadjiev"; all the types with additional: printed (on white paper) "MUSÉUM PARIS | 1934 | COLL H FRUHSTORFER"; lectotype, paralectotype 2 with additional: printed (on red paper) "Type"; lectotype, paralectotype 3 with additional: printed (on white paper) "Velebit Ostraria | M. Hilf $1910 \mid$ O. Leonhard"; paralectotypes 2, 4 with additional: printed (on white paper) "Bosnia 1904 | Korična | O. Leonhard". Although not labelled properly, the lectotype has been designated by Bernardi \& de Lesse (1951: 139). Treated as a junior subjective synonym of Papilio trivia trivia [Denis \& Schiffermüller], 1775 (Higgins, 1941: 269).

leonhardi Fruhstorfer, 1917 (Nymphalidae) "M[elitaea]. cynthia leonhardi subspec. nova" (Fruhstorfer, 1917a: 5). Type locality: "Bulgarien, Rhodope [Rila]" (ibid.: 5). Lectotype $\widehat{O}$, paralectotypes 2 우 with labels: [1 $\hat{\delta}$, lectotype] (1) printed with handwritten inscriptions, here italicised (on white paper) "Bulg. Rhodope Geb. | 30.7. | M. Hilf 1911 | Coll. O. Leonhard"; (2) handwritten (on white paper with black frame) "cynthia | leonhardi | Frhst."; (3) handwritten (on white paper) "Melitaea cynthia $\mid$ ssp. leonhardi Frhst. 1916 (Arch. Nat., A, | 2, p. 3, T . I, fig. 2-3)"; (4) printed (on white paper) "MNHN | $0036 \mid \mathrm{S}$. Abadjiev"; [2 9$]$ (1) printed with handwritten inscriptions, here italicised (on white paper) "Bulg. Rhodope Geb. | 29.7. | M. Hilf 1911 | Coll. O. Leonhard"; (2) handwritten (on white paper) "Melitaea cynthia | ssp. leonhardi Frhst. | 1916 (Arch. Nat., A, 2, p. 3, T . I. fig. 2-3)"; (3) printed (on white paper) "MNHN | 0037 | S. Abadjiev"; [3 +] (1) printed with handwritten inscriptions, here italicised (on white paper) "Bulg. Rhodope Geb. | 22.7. | M. Hilf 1911 | Coll. O. Leonhard"; (2) printed (on white paper) "MNHN | 0052 | S. Abad-
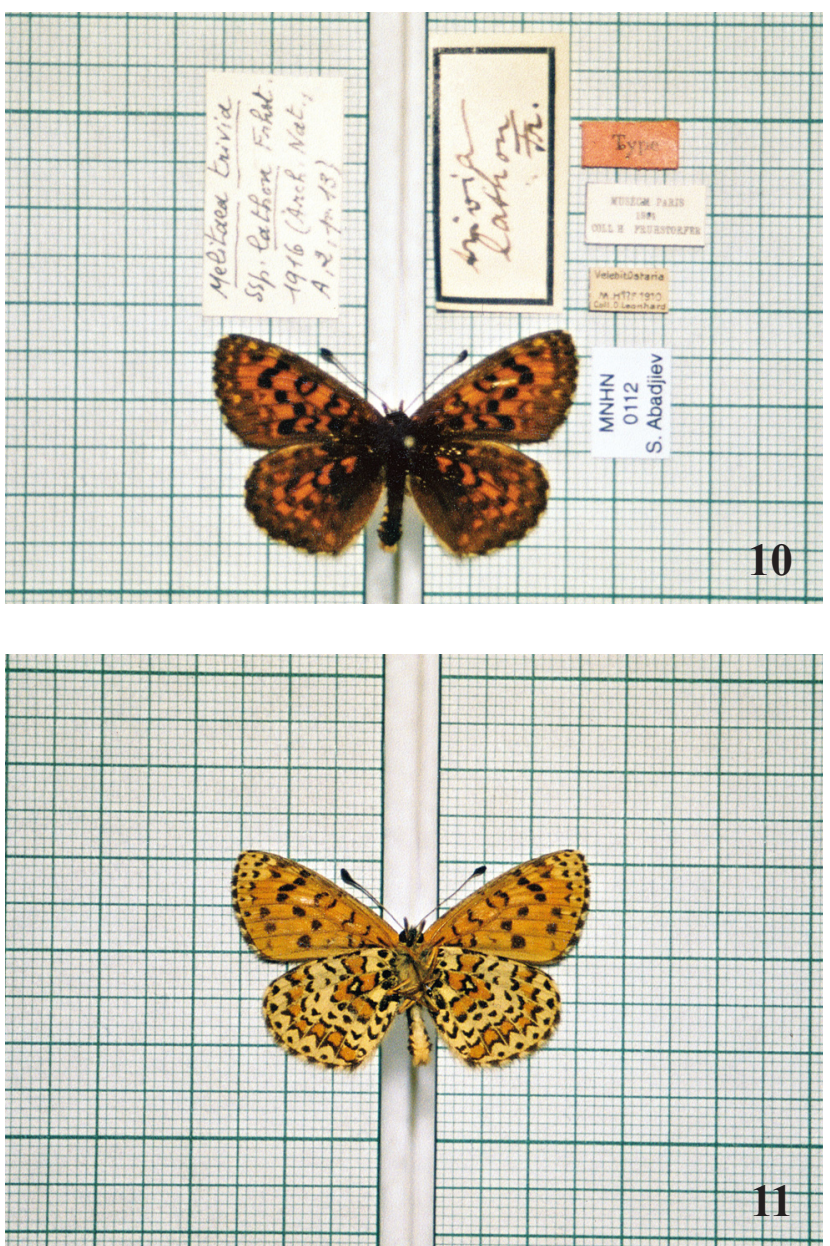

Figs 10-11. [10] Lectotype $\widehat{\jmath}$ of "Melitaea trivia lathon" Fruhstorfer, 1917; [11] ditto, underside.

jiev"; all the types with additional: printed (on white paper) "MUSÉUM PARIS | 1934 | COLL H FRUHSTORFER"; lectotype, paralectotype 2 with additional: printed (on red paper) "Type". Originally the type series comprises $2 \widehat{\delta} \widehat{\delta}, 2$ 웅 (ibid.: 5). The specimens are collected in the current territory of Rila and not in the Rhodopes. Although not labelled properly, lectotype designated by Bernardi \& de Lesse (1951: 143). Treated as a distinct subspecies, Euphydryas cynthia leonhardi (Fruhstorfer, 1917).

lesora Fruhstorfer, 1917 (Nymphalidae) "M[elitaea]. didyma lesora subspec. nova" (Fruhstorfer, 1917a: 8). Type locality: "Krain, Laibach [Ljubljana]" (ibid.: 8). Syntypes $10 \hat{\jmath}, 4$ 우 with labels: [1 §] printed (on white paper) "MNHN $0084 \mid$ S. Abadjiev"; [2 今]] printed (on white paper) "MNHN | 0085 | S. Abadjiev"; [ 3 \] printed (on white paper) "MNHN

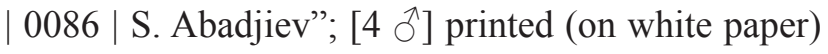


"MNHN | 0087 | S. Abadjiev"; [5 ฏ ] (1) handwritten (on white paper) "lesora"; (2) printed (on white paper) "MNHN | 0088 | S. Abadjiev"; [6 §̋] printed (on white paper) "MNHN | 0089 | S. Abadjiev"; [ 7 今] printed (on white paper) "MNHN | $0090 \mid$ S. Abadjiev"; [ 8 引 $]$ printed (on white paper) "MNHN $0091 \mid$ S. Abad-

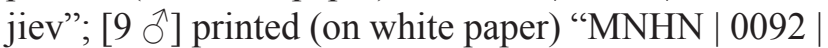
S. Abadjiev"; [10 $\left.{ }^{3}\right]$ (1) handwritten (on white paper with black frame) "didyma | lesora | Fr."; (2) handwritten (on white paper) "Melitaea didyma $\mid$ ssp. lesora Frhst. 1916 (Arch. Nat. A, 2, p. 8)"; (3) printed (on white paper) "MNHN | 0103 | S. Abadjiev"; [11 ?] printed (on white paper) "MNHN | $0093 \mid \mathrm{S}$. Abadjiev"; [12 9 ] printed (on white paper) "MNHN | 0094 | S. Abadjiev"; [13 9] printed (on white paper) "MNHN | 0095 | S. Abadjiev"; [14 9 ] (1) handwritten (on white paper) "Melitaea didyma $\mid$ ssp. lesora Frhst.| 1916 (Arch. Nat. A, | 2, p. 8)"; (2) printed (on white paper) "MNHN | 0104 | S. Abadjiev"; all the syntypes with additional: (1) printed (on white paper, double framed) "Krain | H. Fruhstorfer"; (2) printed (on white paper) "MUSÉUM PARIS | 1934 | COLL H FRUHSTORFER". The originally stated type series comprises $12 \widehat{\partial}, 4$ 우 (ibid.: 8). The lectotype designation by Bernardi \& de Lesse (1951: 139) can be considered invalid since there are 10 o 0 with "Krain |H. Fruhstorfer" and none of them is labelled properly as a lectotype. A junior subjective synonym of Papilio didyma didyma Esper, [1778] (Higgins, 1941: 217).

limera Fruhstorfer, 1917 (Nymphalidae) "M[elitaea $]$. athalia limera subspec. nova" (Fruhstorfer, 1917a: 3). Type locality: "Bosnien, Koricna" (ibid.: 3). Lectotype + , paralectotypes $1 \hat{\jmath}, 2$ 우 $ᄋ$ with labels: [1 $\left.{ }^{\top}\right]$ (1) handwritten (on white paper) "Prép de Leste | n 436"; (2) handwritten (on white paper) "Melitaea athalia $\mid$ ssp. limera Frhst. | 1916 (Arch. Nat. |A, 2, p. 3)"; (3) printed (on white paper) "MNHN 0032 | S. Abadjiev"; [2 9 , lectotype] (1) printed (on red paper) "Type"; (2) printed with handwritten inscriptions, here italicised (on white paper) "MUSÉUM PARIS | figuré | par | Verity"; (3) handwritten (on white paper) "Melitaea athalia | ssp. limera Frhst. | 1916 (Arch. Nat., |A, 2, p. 3)"; (4) printed (on white paper) "MNHN | 0033 | S. Abadjiev"; [3 9 ] printed (on white paper) "MNHN | 0057 | S. Abadjiev"; [4 9 ] printed (on white paper) "MNHN $0058 \mid$ S. Abadjiev"; all the types with additional: (1) printed (on white paper) "Bosnia 1904 | Korična | O. Leonhard"; (2) printed (on white paper) "MUSÉUM PARIS | 1934 | COLL H FRUHSTORFER". Although not labelled properly, lectotype designated by Bernardi \& de Lesse (1951: 142). A junior subjective synonym of Papilio athalia athalia Rottemburg, 1775.

narenta Fruhstorfer, 1917 (Nymphalidae) - "Melitaea phoebe narenta subspec. nova" (Fruhstorfer, 1917a: 1). Type locality: "Herzegowina, Jablanica" (ibid.: 1). Syntypes $\hat{\partial}$, $q$ with labels: [ $\widehat{\jmath}]$ (1) printed (on white paper) "Hercegovina |Cvrstnica|1901"; (2) handwritten (on white paper) "narenta"; (3) printed (on red paper) "Type"; (4) printed (on white paper) "MNHN | 0097 | S. Abadjiev"; [9] (1) printed with handwritten inscriptions, here italicised (on white paper) "Hercegovina | Jablanica | 1901 8.7."; (2) printed (on white paper) "MNHN | $0096 \mid$ S. Abadjiev"; both the syntypes with additional: (1) handwritten (on white paper) "Melitaea phoebe | ssp. narenta Frhst. 1916 (Arch. Nat., A, 2, p. 1)"; (2) printed (on white paper) "MUSÉUM PARIS | 1934 | COLL H FRUHSTORFER". Originally the material mentioned comprises " 5 우 0 in Kollektion Leonhard $\widehat{\partial}$ 우-Type in Kollektion Fruhstorfer" (ibid.: 1). Bernardi \& de Lesse (1951: 141) have incorrectly referred only to a holotype $\hat{\partial}$, since the originally mentioned types are at least 2. Moreover, the specimen labelled "Jablanica" is $Q$ and not $\hat{\partial}$. The other one is labelled after the nearby mountain Čvrsnica. It can not be considered a valid lectotype designation as stated in their introduction and none of the specimens is labelled as such by Bernardi \& de Lesse (1951). Russell \& Tennent (2016: 49) correctly stated that van Oorschot \& Coutsis (2014) wrongly interpreted the type locality as Mount Jablanica, which is on the Macedonia/Albania border. A junior subjective synonym of Papilio phoebe ([Denis \& Schiffermüller], 1775) (van Oorschot \& Coutsis, 2014: 61).

nautaca Fruhstorfer, 1917 (Nymphalidae) - “Argynnis hecate nautaca subspec. nova" (Fruhstorfer, 1917c: 26). Type locality: "Krain, Wippach" (ibid.: 26). Syntypes $\partial$,,+ with labels: [ठ] (1) printed (on red paper) "Type"; (2) printed (on white paper) "MNHN 0113 | S. Abadjiev"; [O] printed (on white paper) "MNHN | 0114 | S. Abadjiev"; both syntypes with additional: (1) printed (on white paper) "L. Karlinger | Coll. e. Wippach | Krain, 20/6 1902"; (2) printed (on white paper) "hecate $\mid$ nautaca $\mid$ Fruhst.". A junior subjective synonym of Papilio hecate hecate [Denis \& Schiffermüller], 1775.

ogygia Fruhstorfer, 1908 (Nymphalidae) - "[Melitaea phoebe] ogygia nov. subspec" (Fruhstorfer, 1908a: 310). Type locality: "Griechenland, Poros" 
(ibid.: 310). Holotype + (by monotypy) with labels: (1) printed (on white paper, framed) "Griechenland Fruhstorfer"; (2) handwritten (on white paper) "Poros 14/6 03"; (3) handwritten (on white paper) "ogygia"; (4) handwritten (on white paper) "Melitaea phoebe ssp. ogygia Frhst. 1907 (Int. Ent. Zeit p. 311)"; (5) printed (on red paper) "Type"; (6) printed (on white paper) "MUSÉUM PARIS | 1934 | COLL H FRUHSTORFER"; (7) printed (on white paper) "MNHN 0098 | S. Abadjiev". Type locality and type status commented by Russell et al. (2007: 140), Russell \& Tennent (2016: 50). Treated as a junior subjective synonym of Melitaea ornata Christoph, 1893 (Russell \& Tennent, 2016: 50).

omotimoius Fruhstorfer, 1921 (Papilionidae) "P[arnassius $]$. apollo omotimoius subspec. nova" (Fruhstorfer, 1921: 87). Type locality: "Vitosch bei

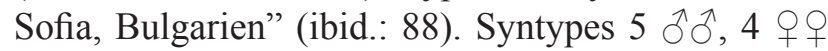
with labels: $\left[\begin{array}{ll}1 & 1\end{array}\right]$ (1) handwritten (on white paper) "omotimoius"; (2) printed (on white paper) "MNHN | 0001 | S. Abadjiev"; [2 §] (1) printed (on white paper) "MNHN | $0002 \mid$ S. Abadjiev"; [3 3$]$ (1) printed (on white paper) "MUSÉUM PARIS"; (2) printed (on white paper with black frame) "Coll. Eisner | Berlin"; (3) printed (on white paper) "MNHN $0003 \mid \mathrm{S}$. Abadjiev"; [ 4 今] printed (on white paper) "MNHN | $0004 \mid$ S. Abadjiev"; [5 3 ] $]$ printed (on white paper) "MNHN 0005 | S. Abadjiev"; [6 o) (1) printed with handwritten inscriptions, here italicised (on white paper) "MUSÉUM PARIS $\mid$ C. Eisner"; (2) printed (on white paper) "685."; (3) printed (on white paper) "46."; (4) printed (on white paper) "O"; (5) printed (on white paper) "MNHN | 0006 | S. Abadjiev"; [7 9] printed (on white paper) "MNHN | $0007 \mid$ S. Abadjiev"; [8 + ] printed (on white paper) "MNHN $|0008|$ S. Abadjiev"; [9 9] (1) printed (on white paper) "1952 | COLL. R. OBERTHÜR"; (2) printed (on white paper) "MNHN | 0009 | S. Abadjiev"; all syntypes with additional: (1) printed (on white paper) "Vitosch | Juli Sofia | Bulgarien"; (2) printed with handwritten inscriptions, here italicised (on white paper) "SYNTYPE $\widehat{\sigma}[q$ respectively] | Parnassius | apollo | omotimoius |Fruhstorfer, $1921 \mid$ S. Abadjiev October, 2003"; syntypes 1, 2, 4, 7, 8 with additional: printed (on white paper) "MUSÉUM PARIS | 1952 | COLL. R. OBERTHÜR"; syntypes 5, 9 with additional: printed (on white paper) "Coll. R. Oberthür $\mid$ Coll. J. Bourgogne $\mid$ Muséum Paris | 1993". Additional syntype 9 known from BMNH (Abadjiev, 2002: 14). A junior subjective synonym of P. apollo graecus Ziegler, 1901 (Abadjiev, 2002: 14).
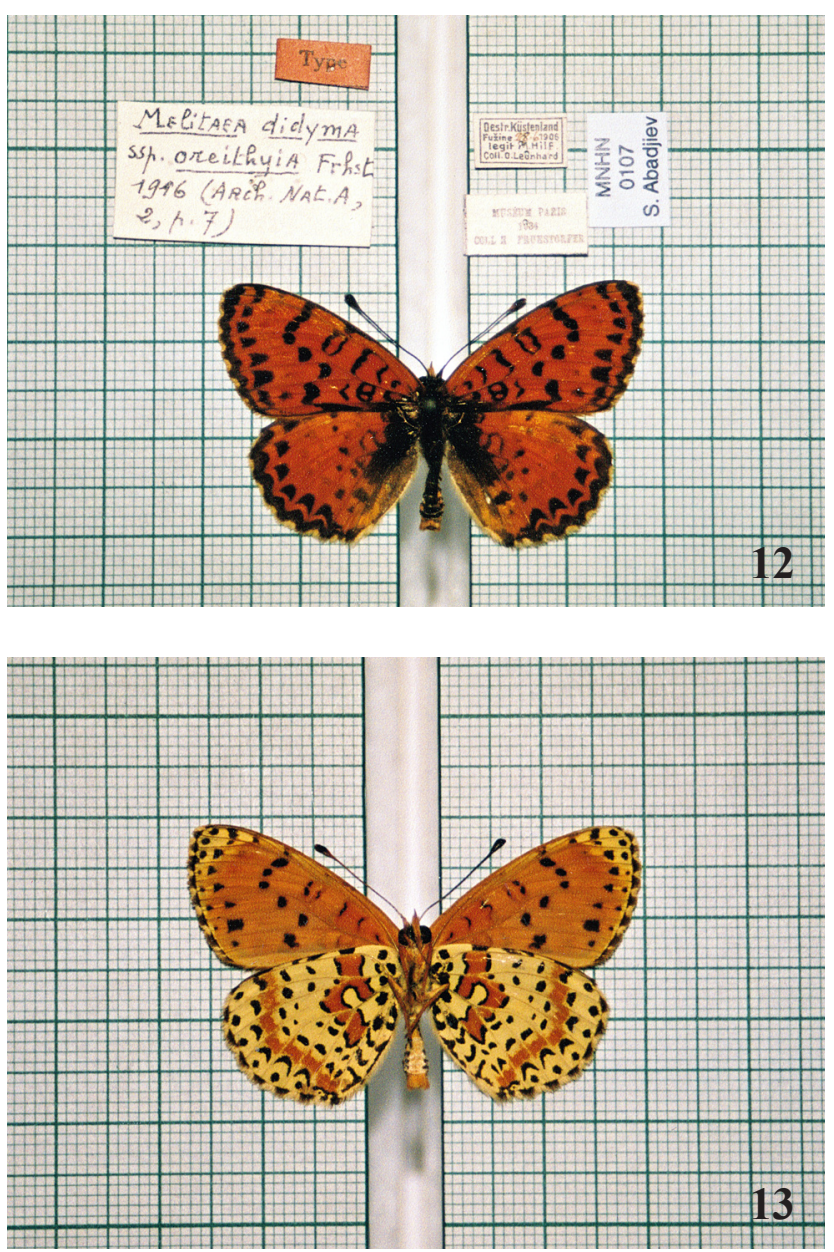

Figs 12-13. [12] Lectotype đ̊ of "Melitaea didyma oreithyia" Fruhstorfer, 1917; [13] ditto, underside.

oreithyia Fruhstorfer, 1917 (Nymphalidae) "M[elitaea $]$. didyma oreithyia subspec. nova" (Fruhstorfer, 1917a: 7). Type locality: "[Croatia] Österr. Küstenland, Fucine" (ibid.: 7). Lectotype $\widehat{\delta}$, paralectotypes $1 \hat{\jmath}, 3$ o 9 with labels: [1 $\left.{ }^{\Uparrow}\right]$ (1) handwritten (on white paper with black frame) "didyma | oreithyia | Fruhstorfer"; (2) printed (on white paper) "MNHN | 0081 | S. Abadjiev"; [2 Ô, lectotype] (Figs 12-13) (1) printed with handwritten inscriptions, here italicised (on white paper with black frame) "Oesterr. Küstenland | Fužine 28.6 1906 | legit M. Hilf | Coll. O. Leonhard"; (2) printed (on white paper) "MNHN | 0107 | S. Abadjiev"; [3 9] (1) printed with handwritten inscriptions, here italicised (on white paper with black frame) "Oesterr. Küstenland | Fužine 16.71906 | legit M. Hilf | Coll. O. Leonhard"; (2) printed (on white paper) "MNHN | 0082 | S. Abadjiev"; [4 + ] printed (on white paper) "MNHN | 0083 | S. Abadjiev"; [5 \&] (1) 
printed with handwritten inscriptions, here italicised (on white paper with black frame) "Oesterr. Küstenland | Fužine 20.71906 | legit M. Hilf | Coll. O. Leonhard"; (2) printed (on red paper) "Type"; (3) printed (on white paper) "MNHN $|0108|$ S. Abadjiev"; all the types with additional: printed (on white paper) "MUSÉUM PARIS | 1934 | COLL H FRUHSTORFER"; paralectotypes 1, 4 with additional: printed with handwritten inscriptions, here italicised (on white paper with black frame) "Oesterr. Küstenland | Fužine 18.6 1906 | legit M. Hilf | Coll. O. Leonhard”; lectotype, paralectotype 5 with additional: handwritten (on white paper) "Melitaea didyma | ssp. oreithyia Frhst. | 1916 (Arch. Nat. A, |2, p. 7)". The originally mentioned type series comprises more than $2 \widehat{\widehat{\partial}}, 3$ 우 (ibid.: 7). Although not labelled properly, lectotype designated by Bernardi \& de Lesse (1951: 139). A junior subjective synonym of Papilio didyma didyma Esper, [1778] (Higgins, 1941: 219).

parnassia Bernardi, 1970 (Pieridae) "A[nthocharis]. gruneri parnassia subsp. nov." (Bernardi, 1970: 2). Type locality: "Graecia, Parnassus" (ibid.: 4). Holotype $\hat{\sigma}$ with labels: (1) printed (on white paper) "37... O Anthocharis | Grüneri | Griechenland | 1833 Müery | 15 Staudinger"; (2) printed (on red paper) "HOLOTYPE"; (3) printed (on white paper) "1923 | coll. Jean SCHLUMBERGER | don de son fils | M. Ernest SCHLUMBERGER | MUSÉUM PARIS"; (4) printed (on white paper) "MNHN | 0029 | S. Abadjiev"; paratypes $9 \widehat{\partial} \widehat{\partial}, 1$ क with labels: [1 \] (1) printed (on white paper) "MUSÉUM PARIS | 1907 | COLL. L. DEMAISON"; (2) printed (on white paper) "MNHN | 0019 | S. Abadjiev"; [2 3$]$ printed (on white paper) "MNHN | $0020 \mid$ S. Abadjiev"; [3 3$]$ printed (on white paper) "MNHN | $0021 \mid$ S. Abadjiev"; [ 4 ○] (1) printed (on white paper) "MUSÉUM PARIS | COLL. D. DUPONT | 1943"; (2) handwritten (on white paper) "Grece | ex Stdgr"; (3) printed (on white paper) "MNHN | $0022 \mid$ S. Abadjiev"; [ 5 ○] printed (on white paper) "MNHN $0023 \mid$ S. Abad-

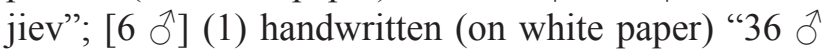
| grüneri v | Thlaspidri | Grece | Dapnisa"; (2) printed (on white paper) "MNHN | $0024 \mid$ S. Abadjiev"; [ 7 引 $]$ printed (on white paper) "MNHN $0025 \mid$ S. Abadjiev"; [ 8 Љै] (1) printed with handwritten inscriptions, here italicised (on white paper) "Mn Hymette | Gréce | $1910 \mid$ Coll. J. Germain | MUSÉUM PARIS”; (2) printed (on white paper) "MNHN | 0026 | S. Abadjiev"; [9 \] (1) printed (on white paper) "Europe meridle."; (2) handwritten (on white paper) "Anthocharis | gruneri |
Neo."; (3) blank (on red paper); (4) printed (on white paper) "1919 | coll. P. THIERRY-MIEG | MUSÉUM PARIS"; (5) printed (on white paper) "MNHN | 0027| S. Abadjiev"; [10 \$] (1) printed (on white paper) "37 + Anthocharis | Grüneri | Griechenland | 1833. Müery | 30 Staudinger"; (2) printed (on white paper) "MNHN | 0028 | S. Abadjiev"; paratypes 1-8, 10 with additional: printed (on red paper) "PARATYPE"; paratypes 2 , 3 with additional: printed with handwritten inscriptions, here italicised (on white paper) "Grece | 1892 | Coll. de Beaulien | MUSÉUM PARIS”; paratypes 1, 6 with additional: handwritten (on white paper) "36 $\sigma^{\lambda}$ | Grece"; paratypes 5, 7 with additional: printed (on white paper) "MUSÉUM PARIS | 1918 | EX. COLL. C. WARD | COLL. R. GALICCHON"; paratypes 6, 10 with additional: printed (on white paper) "1923 | coll. JEAN SCHLUMBERGER | don de son fils | M. Ernest SCHLUMBERGER | MUSÉUM PARIS". Additional $14 \hat{\partial} \widehat{\partial}, 5 q q$ paratypes exist in the BMNH (Abadjiev, 2002: 16-17); also in ZMHB. Currently treated as a junior subjective synonym of Anthocharis gruneri gruneri Herrich-Schäffer, [1851] (Hesselbarth at al., 1995: 362).

praxilla Fruhstorfer, 1917 (Nymphalidae) "M[elitaea]. dictynna praxilla subspec. nova" (Fruhstorfer, 1917a: 13). Type locality: "Krain, Umgebung von Laibach" (ibid.: 14). Syntypes 8 ô, 3 9 + 9 with

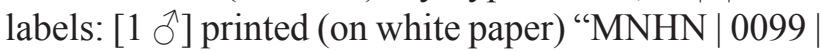
S. Abadjiev"; [ 2 §] printed (on white paper) "MNHN | 0062 | S. Abadjiev"; [3 3 ] printed (on white paper) "MNHN | 0063 | S. Abadjiev"; [4 ठ] printed (on white paper) "MNHN | 0064 | S. Abadjiev"; [5 §] printed (on white paper) "MNHN | $0065 \mid$ S. Abadjiev"; [6 ठ] printed (on white paper) "MNHN $0066 \mid$ S. Abadjiev"; [ $\left.7 \begin{array}{l}7 \\ \text { ] }\end{array}\right]$ printed (on white paper) "MNHN | 0067

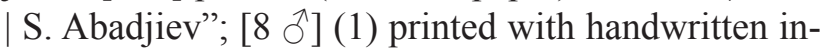
scriptions, here italicised (on white paper with black frame) "dictynna $\mid$ praxilla $\mid$ Fruhst."; (2) printed (on white paper) "MNHN | 0068 | S. Abadjiev"; [9 q] printed (on white paper) "MNHN $0100 \mid$ S. Abadjiev"; [10 +] printed (on white paper) "MNHN $|0069|$ S. Abadjiev"; [11 q] printed (on white paper) "MNHN | 0070 | S. Abadjiev"; syntypes 1, 6 with additional: printed with handwritten inscriptions, here italicised (on white paper) "MUSÉUM PARIS | figuré | par | Verity"; syntypes 1, 9 with additional: (1) handwritten (on white paper) "praxilla"; (2) handwritten (on white paper) "Melitaea dictynna | ssp. praxilla Frhst. | 1916 (Arch. Nat., A, | 2, p. 13)"; (3) printed (on red paper) "Type"; all the syntypes with additional: (1) printed 
(on white paper with double frame) "Krain | Fruhstorfer"; (2) printed (on white paper) "MUSÉUM PARIS | 1934 | COLL H FRUHSTORFER". The lectotype designation by Bernardi \& de Lesse (1951: 140) can be considered invalid since there are 8 oै 0 with "Krain Fruhstorfer" and none of them is labelled properly as a lectotype. A junior subjective synonym of Papilio diamina diamina Lang, 1789 (Higgins, 1941: 323).

scardona Fruhstorfer, 1910 (Nymphalidae) "Mel[itaea]. athalia scardona subspec. nova" (Fruhstorfer, 1910b: 51). Type locality: "Agram [Zagreb]" (ibid.: 51). Syntypes 3 $\hat{\sigma} \widehat{\partial}$ with labels: [1] (1) printed with handwritten inscriptions, here italicised (on white paper) "MUSÉUM PARIS | genitalia 7 | Verity"; (2) handwritten (on white paper) "Melitaea athalia | ssp. scardona Frhst | 1910 (Soc. ent. p. 53)"; (3) handwritten (on white paper) "scardona"; (4) printed (on red paper) "Type"; (5) printed (on white paper) "MNHN | 0030 | S. Abadjiev"; [2] printed (on white paper) "MNHN | 0055 | S. Abadjiev"; [3] (1) handwritten (on white paper with black frame) "athalia $\mid$ scardona | Frhst."; (2) printed (on white paper) "MNHN | 0056 | S. Abadjiev"; syntypes 1, 2 with additional: printed with handwritten inscriptions, here italicised (on white paper) "MUSÉUM PARIS | figuré | par | Verity"; all the syntypes with additional: (1) printed with handwritten inscriptions, here italicised (on yellowish paper) "Agram | Fruhstorfer."; (2) printed (on white paper) "MUSÉUM PARIS | 1934 | COLL. H. FRUHSTORFER". The lectotype designation by Bernardi $\&$ de Lesse (1951: 142) can be considered invalid since there are 3 o $\delta$ with "Agram | Fruhstorfer.", 2 of which with "MUSÉUM PARIS | figuré | par | Verity", and none of these specimens is labelled properly as a lectotype. A junior subjective synonym of Papilio athalia athalia Rottemburg, 1775.

sisenna Fruhstorfer, 1910 (Nymphalidae) - "Argynnis niobe sisenna subspec. nova" (Fruhstorfer, 1910a: 37). Type locality: "Krain, Südtirol, Umgebung von Klausen" (ibid.: 37). Syntype $\widehat{\jmath}$ with labels: (1) printed (on white paper) "Klausen | 1904 | Fruhstorfer"; (2) printed (on white paper) "niobe | sisenna | Fruhst."; (3) printed (on red paper) "Type"; (4) printed (on white paper) "MUSÉUM PARIS | 1934 | COLL H FRUHSTORFER"; (5) printed (on white paper) "MNHN | $0042 \mid$ S. Abadjiev". A junior subjective synonym of Papilio niobe niobe Linnaeus, 1758.

taygetana Bryk \& Eisner, 1939 (Papilionidae) "Parnassius mnemosyne taygetana (subsp. nova)" (Bryk \& Eisner, 1939: 43). Type locality: "Tay-
getos-Gebirge, Griechenland" (ibid.: 43). Paratype ô with labels: (1) printed (on white paper) "Taygetos 18.VI.1926"; (2) handwritten (on white paper) "Taygetos | 18-VI-1926"; (3) printed (on red paper) "PARATYPE"; (4) printed (on red paper) "Paratype"; (5) printed with handwritten inscriptions, here italicised (on white paper) "MUSÉUM PARIS $\mid$ coll. $\mid C$. Eisner"; (6) handwritten (on white paper) "subsp. tayge- | tana | Bryk \& Eis"; (7) printed (on white paper) "MNHN $0018 \mid$ S. Abadjiev". Specimen mentioned by Bernardi \& Viette (1966: 96). A junior subjective synonym of Parnassius mnemosyne caucasia Verity, [1911].

terracina Fruhstorfer, 1917 (Nymphalidae) "M[elitaea $]$. cinxia terracina subspec. nova" (Fruhstorfer, 1917a: 14). Type locality: "Krain, Bosnien"

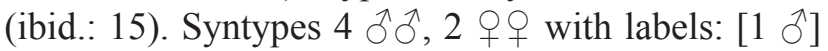
printed (on white paper) "MNHN $0071 \mid$ S. Abadjiev"; [ 2 §ै] printed (on white paper) "MNHN | 0072 | S. Abadjiev"; $\left[\begin{array}{ll}3 & 0\end{array}\right]$ printed (on white paper) "MNHN | 0073 | S. Abadjiev"; [4 O] (1) handwritten (on white paper with black frame) "cinxia | terracina | Frhst"; (2) printed (on white paper) "MNHN $|0101|$ S. Abadjiev"; [5 9] printed (on white paper) "MNHN | $0074 \mid$ S. Abadjiev"; [6 9] printed (on white paper) "MNHN | 0102 | S. Abadjiev"; syntypes 4, 6 with additional: (1) handwritten (on white paper) "Melitaea cinxia | terracina Frhst | 1916 (Arch. Nat. A, 2, p. 14)"; (2) printed (on red paper) "Type"; all the syntypes with additional: (1) printed (on white paper with double black frame) "Krain | H. Fruhstorfer"; (2) printed (on white paper) "MUSÉUM PARIS | 1934 | COLL H FRUHSTORFER". The lectotype designation by Bernardi \& de Lesse (1951: 140) can be considered invalid since there are $4 \hat{\delta} \widehat{\delta}$ with "Krain | H. Fruhstorfer", and none of these specimens is labelled properly as a lectotype. Treated as a junior subjective synonym of Papilio cinxia cinxia Linnaeus, 1758 (Higgins, 1941: 289).

triburniana Fruhstorfer, 1908 (Nymphalidae) "Argynnis hecate triburniana nov. subspec." (Fruhstorfer, 1908b: 214). Type locality: "Herzegowina, Gacko... Vucija-bara... Bosnien, Bocac" (ibid.: 215).

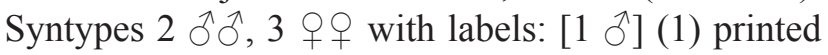
(on white paper) "hecate | triburni- | ana Fruhst."; (2) printed (on white paper) "MNHN $|0038| \mathrm{S}$. Abadjiev"; [2 $\begin{aligned} & 2 \\ & ]\end{aligned}$ (1) printed (on white paper) "Bosnia | Bočac | 3. 7. 08 | DR. SCHAWERDA"; (2) printed (on white paper) "MNHN | $0059 \mid$ S. Abadjiev"; [3 9 ] printed (on white paper) "MNHN | 0043 | S. Abad- 
jiev"; [4 9] printed (on white paper) "MNHN | $0060 \mid$ S. Abadjiev"; [5 9 ] printed (on white paper) "MNHN | 0061 | S. Abadjiev"; syntypes 1, 3 with additional: printed (on white paper) "Hercegovina $\mid$ Gacko | F. v. Meissl"; syntypes 1, 5 with additional: printed (on red paper) "Type"; syntypes 2, 4 with additional: printed (on white paper) "MUSÉUM PARIS | 1934 | COLL H FRUHSTORFER"; syntypes 4, 5 with additional: printed with handwritten inscriptions, here italicised (on white paper with black frame) "Hercegowina | Vucija bara | 19 Juli $07 \mid$ Dr. Schawerda". The originally stated type specimens comprise $2 \hat{\jmath} \hat{\jmath}, 4$ 우 (ibid.: 215). A junior subjective synonym of Papilio hecate hecate [Denis \& Schiffermüller], 1775.

yglanus Fruhstorfer, 1921 (Papilionidae) "P[arnassius]. apollo yglanus subspec. nova" (Fruhstorfer, 1921: 88). Type locality: "Rila Planina, 1400 m, Bulgarien" (ibid.: 88). Syntype $\widehat{\partial}$ with labels: (1) printed with handwritten inscriptions, here italicised (on white paper) "Rila planina $1400 \mathrm{~m}$ ", on the back "18.VII.907"; (2) handwritten (on white paper) "Bulgarien"; (3) handwritten (on white paper) "v. rhodopensis"; (4) printed (on white paper) "MUSÉUM PARIS"; (5) printed (on white paper) "MNHN | 0010 S. Abadjiev"; (6) printed with handwritten inscriptions, here italicised (on white paper) "SYNTYPE 0 | Parnassius | apollo | yglanus | Fruhstorfer, 1921 | S. Abadjiev October, 2003". Treated as a junior subjective synonym of Parnassius apollo graecus Ziegler, 1901 (Abadjiev, 2002: 14). Additional syntype $\bigcirc$ exists in BMNH (Abadjiev, 2002: 14).

\section{Acknowledgments}

The French COLPARSYST (Improving Human Potential Programme of the European Union) provided the funding for this project. Special thanks to my colleagues in the Laboratoire d'Entomologie, Muséum national d'Histoire naturelle, Paris for their invaluable help. I am very obliged to Dr Mario Langourov, National Museum of Natural History, Bulgarian Academy of Sciences, Sofia who kindly assisted with literature.

\section{References}

Abadjiev S. 2001 An annotated list of type specimens of Lepidoptera deposited in the collection of the
Institute of Zoology, Sofia. I. Butterflies (Lep.: Papilionoidea). The Entomologist's Record and Journal of Variation 113: 19-23.

Abadjiev S. 2002 Types of Balkan butterflies in the collection of The Natural History Museum, London (Lepidoptera: Hesperioidea \& Papilionoidea). Neue Entomologische Nachrichten 53: 3-53.

Abadjiev S. 2006 A supplement to "Types of Balkan butterflies in the collection of Natural History Museum, London" (Lepidoptera: Papilionoidea). The Entomologist's Record and Journal of Variation 118: 207-209.

Bernardi G. 1970 La variation géographique de l'Anthocharis gruneri Herrich Schaffer. Lambillionea 70 (1-4): 1-13.

Bernardi G., de Lesse H. 1951 Les types de Nymphalidae paléartiques du Laboratoire d'Entomologie du Museum National d'Histoire Naturelle de Paris. Bulletin de la Société entomologique de France 56: 136-143.

Bernardi G., Viette P. 1966 Les types et typoïdes de Parnassius (s. 1.) se trouvant au Muséum de Paris [Lep. Papilionidae]. Bulletin de la Société entomologique de France 71: 95-100, 229-233.

Bryk F., Eisner C. 1934 Kritische Revision der Gattung Parnassius unter Benutzung des Materials der Kollektion Eisner. Parnassiana 3: 3-22.

Bryk F., Eisner C. 1939 Die griechischen Unterarten von Parn. mnemosyne L. Parnassiana 6: 43-44.

Eisner C. 1936 P. mnemosyne subsp. bulgarica (subsp. nova). Parnassiana 4 (1-2): 9.

Eisner C. 1966 Parnassiidae-Typen in der Sammlung J. C. Eisner. Zoologische Verhandelingen Leiden 81: 1-190, 84 Taf.

Fruhstorfer H. 1908a Neue Argynnis und Melitaea. Internationale entomologische Zeitschrift 1 (41): 310.

Fruhstorfer H. 1908b Neue palaearktische Rhopaloceren. Internationale entomologische Zeitschrift 2 (33): 214-215.

Fruhstorfer H. 1910a Neue paläarktische Argynnisrassen. Entomologische Zeitschrift 24 (7): 37.

Fruhstorfer H. 1910b Neue palaearktische Rhopaloceren. Societas entomologica 25 (13): 49-52.

Fruhstorfer H. 1917a Neue Rhopaloceren aus der Sammlung Leonhard. Archiv für Naturgeschichte. Abteilung A 82 (2): 1-28, 1 Taf.

Fruhstorfer H. 1917b Neue Rhopaloceren aus der Sammlung Leonhard. Societas entomologica 32 (5): 19. 
Fruhstorfer H. 1917c Zwei neue Argynnis Rassen. Societas entomologica 32 (6): 26.

Fruhstorfer H. 1921 Neue Parnassius apollo Rassen. Entomologischer Anzeiger 1 (8): 87-89.

Hesselbarth G., van Oorschot H., Wagener S. 1995 Die Tagfalter der Türkei unter Berücksichtigung der angrenzenden Länder. Selbstverlag Sigbert Wagener, Bocholt, $1354+847$ pp.

Higgins L.G. 1941 An illustrated catalogue of the Palearctic Melitaea (Lep. Rhopalocera). Transactions of the Royal Entomological Society of London 91 (7): 175-365, pl. 1-6.

ICZN 1999 International Code of Zoological Nomenclature. Fourth edition, adopted by the International Union of Biological Sciences. International Trust for Zoological Nomenclature, c/o The Natural History Museum, London, $\mathrm{xxx}+306 \mathrm{pp}$.

Rákosy L., Pecsenye K., Mihali C., Tóth A., Varga Z. 2012 Taxonomic review of Euphydryas maturna (Linnaeus, 1758) (Lepidoptera, Nymphalidae) with description of a new subspecies from Dobrogea (Romania) and notes on conservation biology. Acta Zoologica Academiae Scientiarum Hungaricae 58 (2): 145-161.

Rebel H. 1917 Lepidopteren aus Neumontenegro (Ergebnisse der im Jahre 1916 im Auftrage und auf
Kosten der Kaiserl. Akademie der Wissenschaften in Wien von Dr. Arnold Penther ausgeführten zoologischen Forschungsreise in Serbien und Neumontenegro). Sitzungsberichte der Kaiserlichen Akademie der Wissenschaften. MathematischNaturwissenschaftliche Klasse 126: 765-813.

Russell P., Tennent W.J. 2016 A synonymic list of names associated with western Palaearctic Melitaea phoebe (Denis \& Schiffermüller, 1775) species group taxa (M. phoebe; M. punica Oberthür, 1876; M. ornata Christoph, 1893) (Lepidoptera, Nymphalidae). Nota Lepidopterologica 39 (1): 27-56.

Russell P., Tennent W.J., Pateman J.E., Varga Z.S., Benyamini D., Pe'er G., Bálint Z., Gascoigne-Pees M. 2007 Further investigations into Melitaea telona Fruhstorfer, 1908 (= ogygia Fruhstorfer, 1908, = emipunica Verity, 1919) (Lepidoptera: Nymphalidae), with observations on biology and distribution. Entomologist's Gazette 58: 137-166.

van Oorschot H., Coutsis J.G. 2014 The genus $\mathrm{Me}$ litaea Fabricius, 1807 (Lepidoptera: Nymphalidae, Nymphalinae) Taxonomy and Systematics with special reference to the male genitalia. Tshikolovets, Pardubice, 360 pp. 